\title{
Neonatal mice with necrotizing enterocolitis-like injury develop thrombocytopenia despite increased megakaryopoiesis
}

\author{
Kopperuncholan Namachivayam ', Krishnan MohanKumar', Lalit Garg², Benjamin A. Torres ${ }^{1}$ and Akhil Maheshwari ${ }^{1,3,4}$
}

BACKGROUND: Thrombocytopenia is frequently encountered in infants with necrotizing enterocolitis (NEC). To develop a preclinical model of NEC-related thrombocytopenia, we measured serial platelet counts in 10-d-old (P10) mouse pups with trinitrobenzene sulfonic acid (TNBS)-induced NEC-like injury. We also measured platelet volume indices, immature platelet fraction (IPF), and megakaryocyte number/ploidy in these animals.

METHODS: Platelet counts, platelet volume indices, and IPF were measured in control $(N=65)$ and TNBS-treated pups $(N=$ 104) using an automated hematology analyzer. Bone marrow megakaryocyte number, ploidy and CD41 expression were measured by flow cytometry. These findings were confirmed in a small cohort of P3 mice with NEC-like injury.

RESULTS: Murine pups with TNBS-mediated NEC-like injury developed thrombocytopenia at 15-24h after exposure to TNBS. Intestinal injury was associated with increased platelet volume indices (mean platelet volume, platelet-to-large cell ratio, and platelet distribution width), and IPF, indicating increased thrombopoiesis. These mice also showed increased megakaryocyte number, ploidy, and CD41 expression, indicating increased megakaryocyte differentiation.

CONCLUSION: Similar to human NEC, murine NEC-like injury was also associated with decreased platelet counts. There was evidence of increased megakaryocyte differentiation and thrombopoiesis, which favors peripheral consumption of platelets as the likely mechanism of thrombocytopenia in these animals, over decreased platelet production.

$\mathbf{T}$ hrombocytopenia (platelet counts $<150 \times 10^{9} / 1$ ) is the most frequently encountered hematological abnormality in patients with necrotizing enterocolitis (NEC), seen in 50-95\% of all patients within $24-72 \mathrm{~h}$ of receiving a diagnosis of NEC (1-4). Many infants with severe NEC may have platelet counts in the range of $30-60 \times 10^{9} / 1(1-3)$. The severity of thrombocytopenia generally correlates with Bell's clinical stage of NEC, and some clinical studies suggest that a rapid drop in platelet counts to $<100 \times 10^{9} / 1$ may herald the onset of bacteremia or bowel gangrene $(1,5)$. Platelet counts may also provide important predictive information for the outcome; severe thrombocytopenia $\left(<50 \times 10^{9} / 1\right)$ may predict the need for surgical intervention, NEC-related gastrointestinal complications such as cholestatic liver disease and short bowel syndrome, prolonged length of hospital stay, and mortality $(2,3,5-7)$.

The mechanism of thrombocytopenia in NEC is unclear. There is indirect, anecdotal evidence for platelet consumption, seen in the rapid drop in platelet counts in many patients with NEC and in the short-lived rise in platelet concentrations following transfusions (8). However, there is conflicting data from at least two studies that suggest that some premature infants with sepsis or NEC may have suppressed megakaryopoiesis as the sole or contributing cause of thrombocytopenia $(9,10)$. To understand the mechanism(s) of NEC-related thrombocytopenia, there is a need for robust, developmentally-appropriate preclinical models. In the present study, we sought to address this gap by investigating the temporal evolution of thrombocytopenia in our previously-described neonatal mouse model of trinitrobenzene sulfonic acid-induced NEC-like injury (1113). To determine the kinetic basis of thrombocytopenia during NEC-like injury, we also measured platelet volume indices, immature platelet fraction, and megakaryocyte number/ploidy in the bone marrow of these animals.

\section{METHODS}

Murine Neonatal TNBS-Mediated Enterocolitis

Animal studies were approved by the Institutional Animal Care and Use Committee at University of South Florida. As described previously (11), TNBS enterocolitis was induced in 10-d-old C57BL/6 mice by administering TNBS (2 equal doses of $50 \mathrm{mg} / \mathrm{kg}$ dissolved in $30 \%$ ethanol, w/v, by gavage and rectal instillation, respectively. Control animals $(n=10)$ received vehicle alone. In some experiments, we also compared a small number of P3 mice in control and TNBStreated groups. In all experiments, animals were monitored every $3 \mathrm{~h}$ for physical distress and were euthanized if they developed signs of illness or at $48 \mathrm{~h}$ using $\mathrm{CO}_{2}$ inhalation followed by cervical dislocation. Intestinal injury was confirmed by histopathological analysis of ileocolic region (11): (i) mild injury: disruption of villus tips or mild separation of lamina propria in the small intestine; low level leukocyte

'Department of Pediatrics, Morsani College of Medicine, University of South Florida, Tampa, Florida; ${ }^{2}$ Faculty of Information and Communication Technology, University of Malta, Msida, Malta; ${ }^{3}$ Department of Molecular Medicine, Morsani College of Medicine, University of South Florida, Tampa, Florida; ${ }^{4}$ Department of Community and Family Health, College of Public Health, University of South Florida, Tampa, Florida. Correspondence: Akhil Maheshwari (akhilm@health.usf.edu)

Received 13 September 2016; accepted 19 November 2016; advance online publication 1 March 2017. doi:10.1038/pr.2017.7 
infiltration in the colon in $<10 \%$ high-power fields (HPF) with no structural changes; (ii) moderate injury: mid-villus disruption, clear separation of lamina propria and/or edema in the ileal submucosa; prominent leukocyte infiltration in colon in up to 50\% HPF, crypt elongation, mucosal thickening, superficial ulcerations; (iii) severe injury: transmural injury in the small intestine; marked leukocyte infiltration in $>50 \% \mathrm{HPF}$, elongated and distorted crypts, bowel-wall thickening, and extensive ulcerations.

\section{Platelet Counts and Platelet Volume Indices}

Blood was obtained from mouse pups by puncturing the anterior facial vein with a 25-gauge hypodermic needle (BD Biosciences, San Diego, CA) and was collected using pipette tips and eppendorf tubes flushed with acid-citrate-dextrose buffer (Sigma, St. Louis, MO). Five microliters of blood were added to $95 \mu$ l Cellpak reagent (Sysmex Corporation, Kobe, Japan) and gently mixed to avoid platelet activation. Platelet counts and indices were measured using the Sysmex XT-2000iV automated veterinary hematology analyzer (Sysmex). This instrument measures platelets by both impedance and fluorescence optical methods, and computes the platelet volume indices (MPV, plateletcrit, PDW, and P-LCR) and the IPF. Although this analyzer was originally standardized with $85 \mu \mathrm{l}$ blood volumes, measurements in blood samples diluted 1:20 remain reproducible and accurate (14). To avoid the inadvertent effects of blood loss on intestinal injury and platelet kinetics, we tried to limit the number of samples from each animal to a maximum of 3-4 time-points.

\section{Megakaryocytes}

Bone marrow cells were harvested from both femurs of control and TNBS-treated 10-d-old C57BL/6 mice $(n=10$ each) in Iscove's modified Dulbecco's medium supplemented with $1 \%$ penicillin/streptomycin, $1 \%$ L-glutamine, and 10\% FBS (Life Technologies, Carlsbad, CA) (15). Bone marrow from a few adult mice (9- to 12-wk-old) was used for comparison. Red cells were lysed using hypotonic buffer $(150 \mathrm{mmol} / \mathrm{l} \mathrm{NaCl}, 10 \mathrm{mmol} / \mathrm{l}$ $\left.\mathrm{NaHCO}_{3}, 100 \mathrm{mmol} / \mathrm{lEDTA}\right) \times 10 \mathrm{~min}$ at room temperature. Cells were then labeled using monoclonal FITC- or PE-conjugated anti-CD41 antibody (BD Biosciences) and purified using anti-FITC or PE ferromagnetic microbeads (Miltenyi Biotec, San Diego, CA). Mature megakaryocytes were enriched on a discontinuous density gradient of bovine serum albumin (BSA) fraction $V(0,1.5$, and $3.0 \%$ in phosphate-buffered saline; BSA from Sigma) (16). After $30 \mathrm{~min}$, cells that settled at the bottom of the tube by gravity were harvested and enumerated using an automated counter (model TC20, Bio-Rad, Hercules, CA). Cytospin preparations were stained with the Wright-Giemsa stain, and morphometric measurements were made using the software program Image J (17). Cellular ploidy (after propidium iodide staining) and CD41 expression were measured by flow cytometry (model Accuri C6 plus, BD Biosciences).

\section{Statistical Methods}

Measures of central tendency were computed using IBM SPSS for Windows, version 23 (IBM Corporation, Armonk, NY). Platelet counts, platelet volume indices, IPF, and megakaryocyte indices were evaluated by nonparametric methods. Platelet counts were analyzed as time-dependent covariates in a Cox proportional hazard regression model (18) of time-to-intestinal injury (none, mild, moderate, or severe). Multiple groups were compared by independent samples Kruskal-Wallis $H$ test (19) and the Jonckheere-Terpstra (J-T) test for ordered alternatives (20). The J-T test provides more statistical power than the Kruskal-Wallis test when there is an a priori ordering of the populations, and was, therefore, preferred for comparisons across subgroups defined by severity of intestinal injury or time elapsed in the NEC protocol. Serial IPF measurements were compared by Friedman's test (21). Megakaryocyte indices were evaluated by independent samples Mann-Whitney U-test (22). Numeric data were depicted as Tukey-Koopman box-whisker plots (23), where the median was shown as a bold line, the box showed the interquartile range, and the whiskers showed the minima and maxima. Outliers $(>1.5 \times \mathrm{IQR})$ were depicted as small circles. All statistical tests were two-sided and considered significant at $P<0.05$.

\section{RESULTS}

\section{Blood Platelet Concentrations in TNBS-Mediated Murine NEC-}

\section{Like Injury}

We compared serial platelet counts in TNBS-mediated murine NEC-like injury vs. control animals ( $N=104$ in TNBS-treated and 65 in control group). After TNBS exposure, animals were monitored at regular intervals and were euthanized upon the development of signs of physical distress, or at $48 \mathrm{~h}$. Intestinal injury was evaluated histopathologically at the time of euthanasia and was graded as mild, moderate, and severe in 17, 35 , and 52 mice, respectively. Twenty-one of 35 pups with moderate injury and 45/52 pups with severe intestinal injury had to be euthanized before the completion of the $48 \mathrm{~h}$ study period in view of physical distress. As seen in Figure 1, TNBSmediated NEC-like injury was associated with decreased platelet counts. In pups with mild intestinal injury, platelet counts dropped below baseline at $18 \mathrm{~h}$ and reached a nadir at $24 \mathrm{~h}$. In moderate-severe injury, platelet counts started dropping at $15 \mathrm{~h}$, reached a nadir at $18 \mathrm{~h}(P<0.001$ at both timepoints), and then showed some recovery at $24-48 \mathrm{~h}(P<0.05)$. Interestingly, a transient rise in platelet counts was noted at the $6 \mathrm{~h}$ time-point in severe intestinal injury. Further analysis in Cox regression models confirmed that the association of platelet counts with murine NEC-like injury was time-dependent (hazard ratio $=0.996,95 \%$ confidence interval $0.995-0.997, P$ $<0.001)$. The platelet counts at various time-points are summarized in Table 1.

\section{Platelet Volume Indices in TNBS-Mediated Murine NEC-Like Injury}

To understand the kinetic basis of thrombocytopenia in NEClike injury, we compared platelet volume indices in the control and NEC-like injury groups. The mean platelet volume (MPV) is the quotient of the plateletcrit (ratio of platelet volume to the whole blood volume) and the platelet counts (MPV = (plateletcrit $(\%) /$ platelet count $\left.\left.\left(\times 10^{9} / \mathrm{L}\right)\right) \times 10^{5}\right)$. The Sysmex hematology analyzer reads platelet distribution width (PDW) as the range of platelet volumes at $20 \%$ frequency (peak of the frequency histogram $=100 \%$ ) and the platelet-large cell ratio (P-LCR) is the proportion of large platelets $(>12 \mathrm{fL})$ in the total platelet population (Figure $2 \mathrm{a}$ ).

The platelet volume indices in control and TNBS-treated groups are summarized in Table 2. At the $18 \mathrm{~h}$ time-point, the median MPV in the control group was $6.6 \mathrm{fL}$ (range 6.1-8.3) and increased to 7.5 (6.6-7.7), 7.2 (6.5-9.6), and 7.6 (6.4-9.9) $\mathrm{fL}$ in mice with mild, moderate, and severe intestinal injury, respectively $(P<0.001$; Figure $2 \mathrm{~b})$. MPV rose in moderatesevere intestinal injury occurred despite lower plateletcrits $(P$ $<0.001$; not depicted) due to the concomitant drop in platelet counts. The PDW and P-LCR also increased in pups with intestinal injury $(P<0.001$; Figure $2 \mathrm{c}, \mathrm{d})$. The increase in platelet volume indices in murine NEC-like injury indicated an increased number of larger, presumably younger platelets in the circulation. 


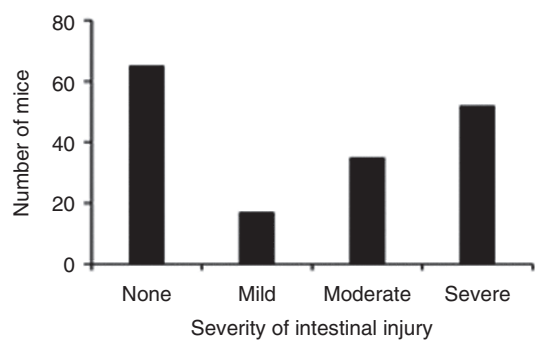

a

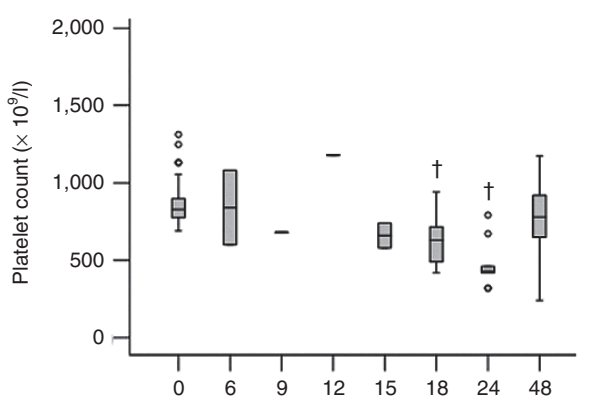

b

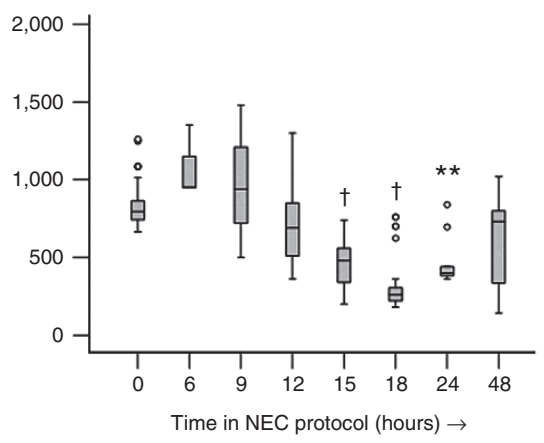

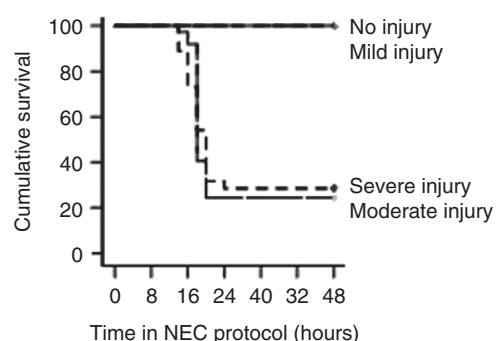

C

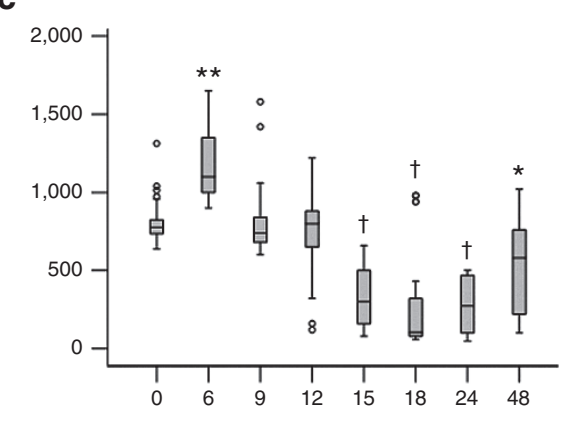

Figure 1. Blood platelet concentrations in TNBS-mediated murine NEC-like injury. Boxplots show blood platelet concentrations in C57BL/6 pups on P10 with (a) mild, (b) moderate, and (c) severe intestinal injury at various time-points between 0 and $48 \mathrm{~h}$ following administration of TNBS. Control mice received vehicle (ethanol) alone and did not show significant changes in platelet counts during the study period. $N=104$ mice with TNBS-mediated NEClike injury and 65 controls. Left inset: Bar diagram shows the distribution of TNBS-treated mice by severity of intestinal injury at the time of euthanasia. Right inset: Kaplan-Meier survival curves show that all mice in control group and with mild injury completed the $48 \mathrm{~h}$ study period, but $21 / 28$ pups with moderate injury and $45 / 63$ with severe intestinal injury had to be euthanized at earlier time-points in view of physical distress. Jonckheere-Terpstra test for ordered alternatives; ${ }^{*} P<0.05,{ }^{* *} P<0.01, \dagger$ (dagger) $P<0.001$ vs. control.

Table 1. Platelet counts in murine TNBS-mediated NEC-like injury

\begin{tabular}{|c|c|c|c|c|c|c|c|c|}
\hline \multirow[b]{2}{*}{ Time-points } & \multicolumn{2}{|r|}{ Controls } & \multicolumn{2}{|c|}{ Mild intestinal injury } & \multicolumn{2}{|c|}{ Moderate intestinal injury } & \multicolumn{2}{|c|}{ Severe intestinal injury } \\
\hline & $N$ & $\begin{array}{l}\text { Platelet counts } \\
\quad(\text { median } \\
(\text { range })) \times 10^{9} / /\end{array}$ & $N$ & $\begin{array}{l}\text { Platelet counts } \\
\quad(\text { median } \\
(\text { range })) \times 10^{9} / \mathrm{l}\end{array}$ & $N$ & $\begin{array}{l}\text { Platelet counts } \\
\quad(\text { median } \\
(\text { range })) \times 10^{9} / \text { I }\end{array}$ & $N$ & $\begin{array}{l}\text { Platelet counts (median } \\
(\text { range })) \times 10^{9} / I\end{array}$ \\
\hline $\mathrm{Oh}$ & 65 & $816(640-1,272)$ & 17 & $827(691-1,313)$ & 35 & $794(664-1,291)$ & 52 & $776(637-1,313)$ \\
\hline $6 \mathrm{~h}$ & 12 & $806(660-1,180)$ & 2 & $840(691-1,313)$ & 3 & $950(950-1,350)$ & 5 & $1100(900-1,650)$ \\
\hline $9 \mathrm{~h}$ & 17 & $880(630-1,118)$ & 1 & $827(600-1,080)$ & 3 & $940(500-1,480)$ & 22 & $740(600-1,580)$ \\
\hline $15 \mathrm{~h}$ & 20 & $783(740-948)$ & 2 & $660(508-740)$ & 31 & $480(200-740)$ & 45 & $300(80-660)$ \\
\hline $18 \mathrm{~h}$ & 16 & $816(640-864)$ & 16 & $630(420-940)$ & 31 & $260(180-760)$ & 46 & $105(60-980)$ \\
\hline $24 \mathrm{~h}$ & 16 & 832 (704-936) & 16 & $430(320-792)$ & 9 & $400(360-840)$ & 15 & $272(48-500)$ \\
\hline $48 \mathrm{~h}$ & 17 & $824(640-1,274)$ & 17 & $780(240-1,176)$ & 12 & $730(140-1,020)$ & 15 & $580(100-1,020)$ \\
\hline
\end{tabular}

NEC, necrotizing enterocolitis; TNBS, trinitrobenzene sulfonic acid.

\section{Circulating Immature Platelet Fraction in TNBS-Mediated Murine NEC-Like Injury}

We next compared the concentration of immature platelets in blood samples from control and TNBS-treated mice. The Sysmex analyzer enumerates immature platelets in the fluorescence channel using a proprietary stain containing the polymethine and oxazine dyes, where immature platelets are identified based on larger size (forward light scatter) and greater nucleic acid content (side fluorescence intensity; Figure 3a) (24). The number of immature platelets in a given blood sample was obtained using previously-described gating parameters (25). At the $18 \mathrm{~h}$ time-point, the median concentration of immature platelets was $46 \times 10^{9} / 1$ in control and decreased to $47.4,28.8$, and $12.4 \times 10^{9} / 1$ in mild, moderate and severe injury, respectively $(P<0.001$; Table 2$)$. However, the immature platelet fraction (proportion of immature platelets in the total platelet population, IPF) increased from $7.4 \%$ (range 2.9-11.9) in control animals to $9.4 \%$ (range 3.1-33.5; $P<0.001$; Figure $3 \mathbf{b}$ ). The severity of intestinal injury did not affect IPF (Table 2). To understand the temporal evolution of IPF during intestinal injury, we obtained serial blood samples from a small cohort of control and TNBS-treated pups ( $n=10$ /group). As seen in Figure $3 c$, the IPFs started rising within the first few hours of TNBS exposure and peaked at $18 \mathrm{~h}(P<0.01)$. We interpreted 


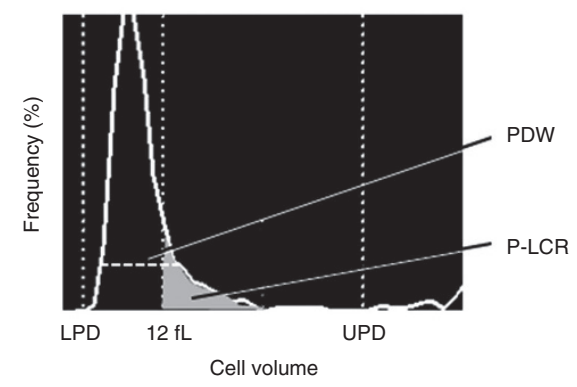

C

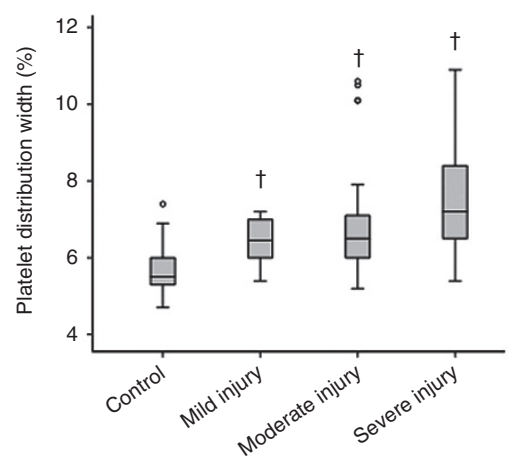

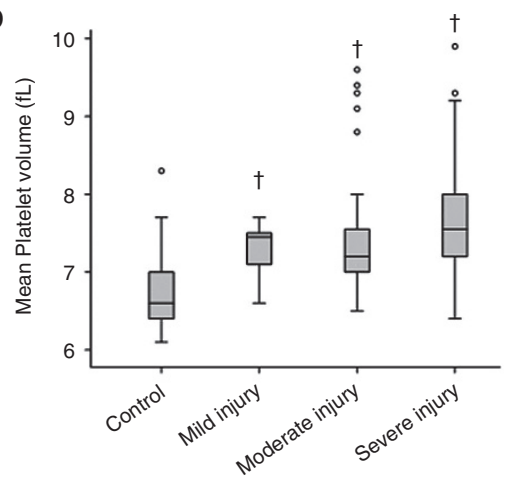

d

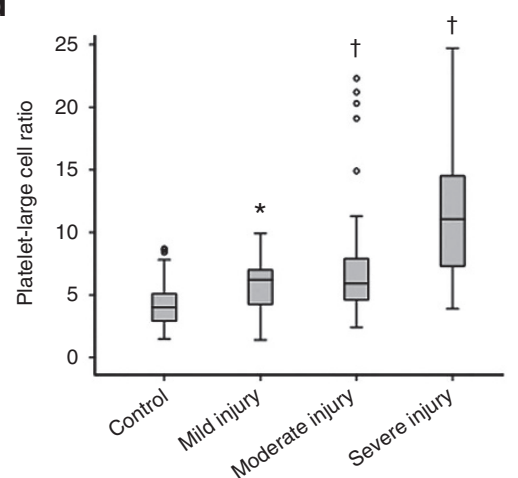

Figure 2. Platelet volume indices in TNBS-mediated murine NEC-like injury. (a) Frequency histogram shows the distribution of platelets by cell volume, generated by the Sysmex XT-2000iV automated veterinary hematology analyzer. The instrument identifies platelets between the lower platelet discriminator (LPD, typically $2 \mathrm{fL}$ ) and the upper platelet discriminator (UPD, typically identified by the instrument between 12-30 fL). Platelet distribution width $(P D W)$ is defined as the range of platelet volumes at $20 \%$ frequency (peak of the frequency histogram $=100 \%$ ). Platelet-large cell ratio $(P-L C R)$ is the proportion of large platelets $(>12 \mathrm{fL})$ in the total platelet population; $(\mathbf{b}-\mathbf{d})$ Boxplots show platelet volume indices in P10 mouse pups in the control vs. NEC-like injury groups with mild, moderate, and severe intestinal injury at the $18 \mathrm{~h}$ time-point following TNBS exposure: panel (b) mean platelet volumes, (c) PDW, and (d) P-LCR. N $=93$ mice with TNBS-mediated NEC-like injury and 65 controls. Jonckheere-Terpstra test for ordered alternatives; ${ }^{*} P<0.05,+P<$ 0.001 vs. control.

Table 2. Platelet volume indices and immature platelets in TNBS-mediated NEC-like injury in P10 pups

\begin{tabular}{|c|c|c|c|c|}
\hline & $\begin{array}{l}\text { Control } \\
(N=65)\end{array}$ & $\begin{array}{l}\text { Mild intestinal } \\
\text { injury }(N=16)\end{array}$ & $\begin{array}{l}\text { Moderate intestinal } \\
\text { injury }(N=31)\end{array}$ & $\begin{array}{c}\text { Severe intestinal } \\
\text { injury }(N=46)\end{array}$ \\
\hline Mean platelet volume (fL; median (range)) & $6.6(6.1-8.3)$ & $7.5(6.6-7.7)$ & $7.2(6.5-9.6)$ & $7.6(6.4-9.9)$ \\
\hline Platelet distribution width (\%; median (range)) & $5.5(4.7-7.4)$ & $6.5(5.4-7.2)$ & $6.5(5.2-10.6)$ & $7.2(5.4-10.9)$ \\
\hline Platelet-large cell ratio (median (range)) & $4(1.5-8.7)$ & $6.2(1.4-9.9)$ & $5.9(2.4-22.3)$ & $11.1(3.9-24.7)$ \\
\hline
\end{tabular}

NEC, necrotizing enterocolitis; TNBS, trinitrobenzene sulfonic acid.

the rise in IPF during NEC-like injury to indicate ongoing thrombopoiesis and next examined megakaryocytes in the bone marrow.

\section{Megakaryocyte Number and Ploidy in the Bone Marrow of Mice With TNBS-Mediated NEC-Like Injury}

We next examined bone marrow megakaryocytes from mice with intestinal injury and control at the $18 \mathrm{~h}$ time-point after exposure to TNBS ( $n=10$ mice/group). As seen in Figure 4a, murine NEC-like injury was associated with increased number of megakaryocytes in the bone marrow. Similar to human infants (26), megakaryocytes in the neonatal murine bone marrow were considerably smaller in size than those in adult mice (Figure $4 \mathrm{~b}$; median area of bone marrow megakaryocytes in pups $=4,749$ vs. $8,906 \mu \mathrm{m}^{2}$ in adult mice, $n=10$ cells/ group, $P<0.001)$. There was no significant difference in the size of bone marrow megakaryocytes from control vs. TNBStreated animals, although there was greater variability in size in the latter. Megakaryocytes from mice with NEC-like injury showed increased ploidy (cells $\geq 8 \mathrm{~N}$ : median 14.8 , range $12.4-$ 18.2 in control vs. 30.9 , range $18.1-36.4$ in NEC-like injury, $P<0.001$; Figure 4c) and CD41 expression (median 61.5\%, 

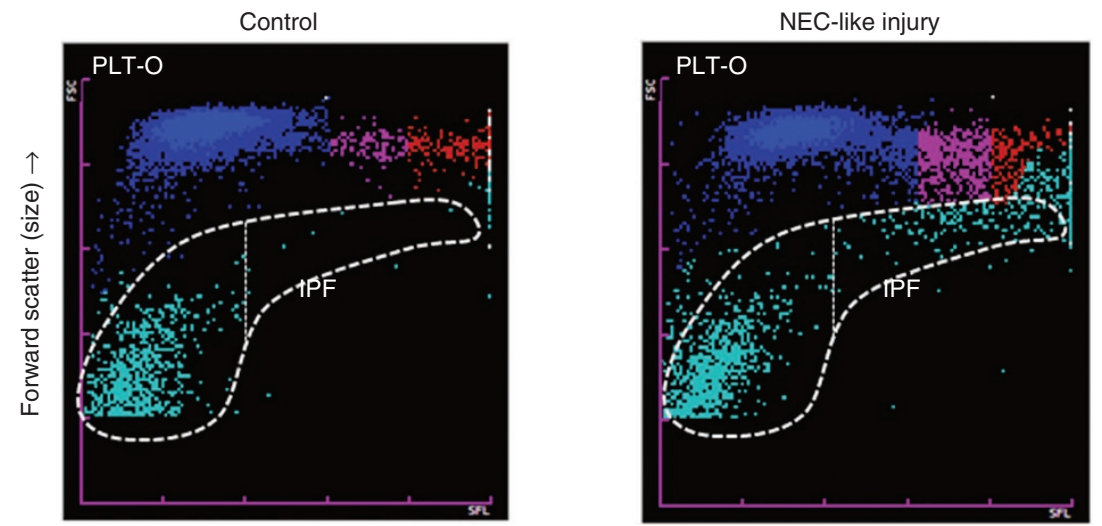

Fluorescence intensity (nucleic acid content)

b

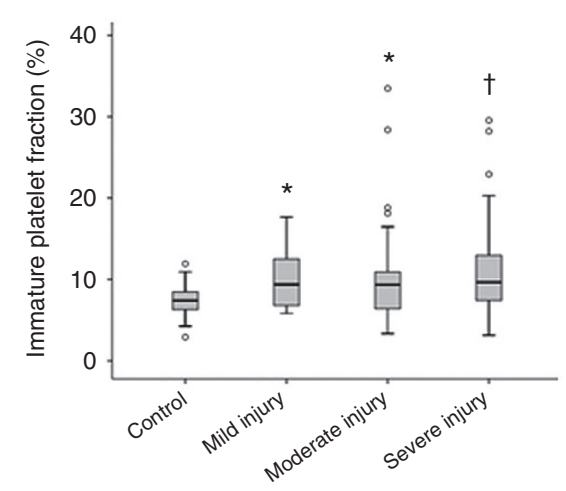

C

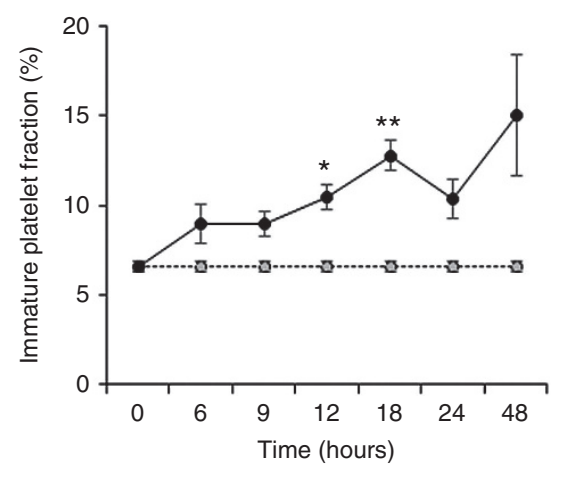

Figure 3. Circulating immature platelet fraction (IPF) in TNBS-mediated murine NEC-like injury. (a) Representative scatter grams from control and TNBStreated pups show increased immature platelets in the IPF gate. (b) Boxplots show the IPF in blood samples from P10 mouse pups in the control vs. NEC-like injury groups with mild, moderate, and severe intestinal injury at the $18 \mathrm{~h}$ time-point following TNBS exposure. $N=93$ mice with TNBS-mediated NEC-like injury and 65 controls. Jonckheere-Terpstra test for ordered alternatives; ${ }^{*} P<0.05,+P<0.001$. (c) Serial measurements in a small cohort of TNBStreated mice show the longitudinal change in IPF during NEC-like injury. $N=10$ mice/group. Friedman's test for repeated measurements; ${ }^{*} P<0.05$, ${ }^{* *} P<$ 0.01 vs. control.

range $52-68 \%$ in control vs. $84 \%$, range $78-92 \%$ in NEC-like injury, $P<0.001$; Figure 4 d). Taken together, these findings indicated increased megakaryocyte commitment/differentiation in NEC-like injury.

\section{Platelet Counts and IPF in P3 Mice With TNBS-Mediated NEC-Like Injury}

The use of mouse pups on postnatal day 10 (P10) to study NEC-like injury is acceptable because the murine neonatal intestine resembles the human midgestation intestine until P14-16 (27,28). However, the transition from fetal (hepatic) to adult (bone marrow) megakaryopoiesis may occur between P5 and P14 (14). In view of this developmental asynchrony between the two organ systems, we sought to validate our findings by measuring platelet counts, platelet volume indices, and IPF in a small cohort of P3 mice with TNBS-mediated intestinal injury. Because of the size-limitation of P3 animals, a single blood sample was obtained at the time of euthanasia at $18 \mathrm{~h}$.

Similar to P10 animals, P3 mice with TNBS-mediated NEClike injury also developed thrombocytopenia (median 328 (range $212-676) \times 10^{9} / \mathrm{L}$ in control vs. 106 (range $\left.24-148\right) \times$ $10^{9} / 1$ in NEC-like injury, $P<0.001$; Figure 5a). The plateletcrit dropped from $0.28 \%$ (range $0.16-0.52$ ) in control to $0.16 \%$ $(0.04-0.2)$ in intestinal injury $(P=0.009)$. The concentration of immature platelets decreased from median 22 (range 18.8$47.2) \times 10^{9} / 1$ in control to $9.4(0.6-25.4) \times 10^{9} / 1$ in intestinal injury, $P=0.003$, but the IPFs were significantly higher $(8.2 \%$, range $6.15-14.4$ in control vs. $13.8 \%$, range $9-17.7$ in intestinal injury, $P=0.03$; Figure 5b).

\section{DISCUSSION}

We present a detailed investigation of the temporal evolution and kinetic mechanisms of thrombocytopenia in a murine neonatal model of NEC-like intestinal injury. The severity of thrombocytopenia in our murine model correlated strongly with injury grade, which is consistent with the inverse correlation between platelet counts and Bell's clinical stage of NEC $(1-3,5,6)$. The onset of thrombocytopenia between 15 and $18 \mathrm{~h}$ is also consistent with the typical course of thrombocytopenia in infants with NEC $(1,5)$. To our knowledge, this is the first report of thrombocytopenia in a preclinical model of NEC.

We have previously described the use of TNBS to induce acute necrotizing enterocolitis in mouse pups (11). The incidence of NEC peaks in premature infants at a post-menstrual 


\section{Articles | Namachivayam et al.}

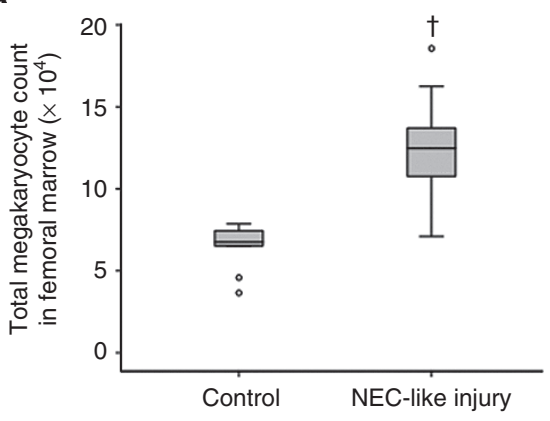

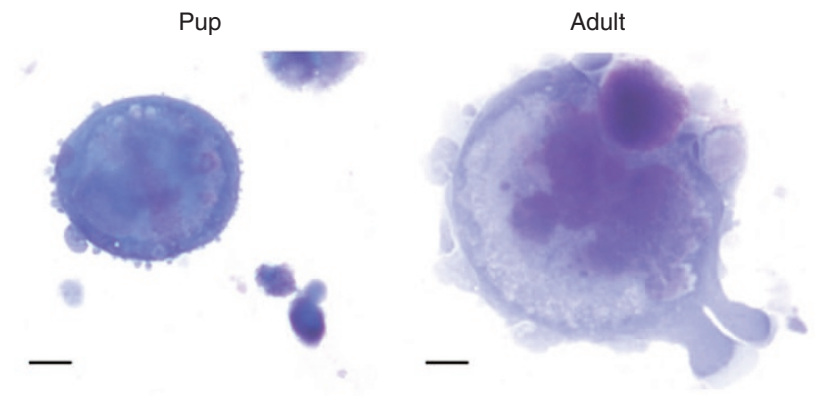

c

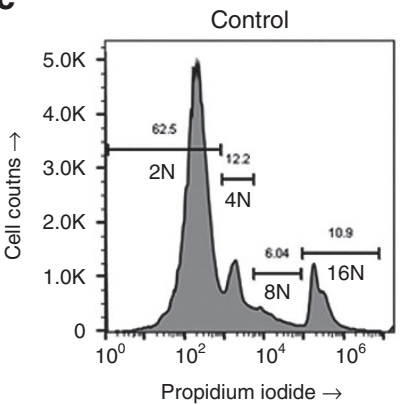

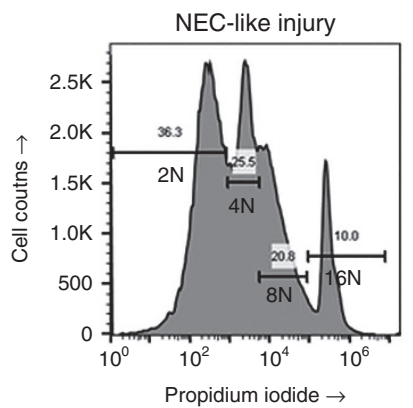

d

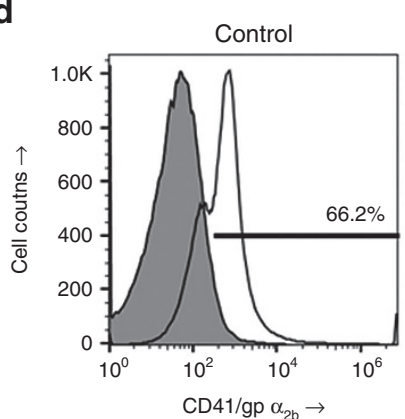

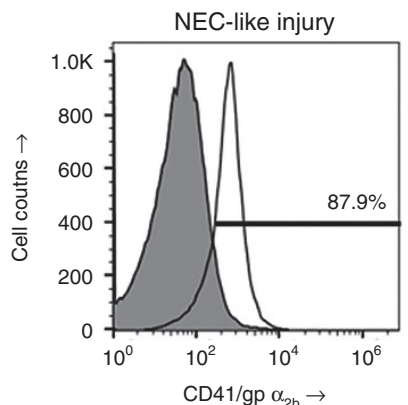

Figure 4. Megakaryocyte number and ploidy in the bone marrow of mice with TNBS-mediated NEC-like injury. (a) Boxplots show megakaryocyte counts in the femoral bone marrow of control and NEC-like injury groups, euthanized $18 \mathrm{~h}$ after TNBS exposure. $N=10$ mice/group. Mann-Whitney $U$-test; $† P<$ 0.001 vs. control. (b) Megakaryocytes from mouse pups on P10 and adult animals, stained with the Wright-Giemsa stain. Magnification $=400 \times$. Scale bar $=10 \mu \mathrm{m}$. (c) Histograms show the increase in megakaryocyte ploidy during NEC-like injury. FACS histograms were obtained on bone marrow megakaryocytes after staining with propidium iodide. Data representative of 10 mice/group. (d) FACS histograms show the increase in CD41 (platelet glycoprotein $\alpha_{2 b}$ ) expression on bone marrow megakaryocytes during NEC-like injury. Data are representative of 10 mice/group.

a

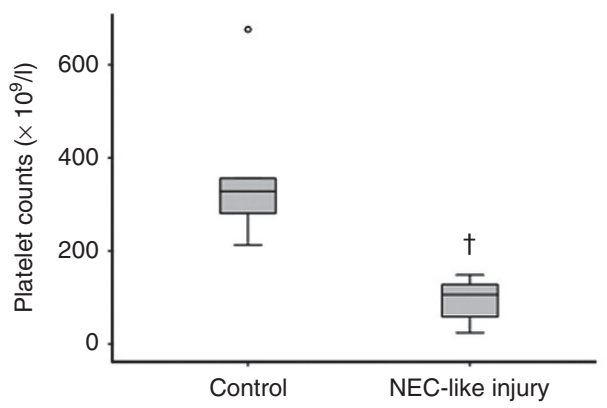

b

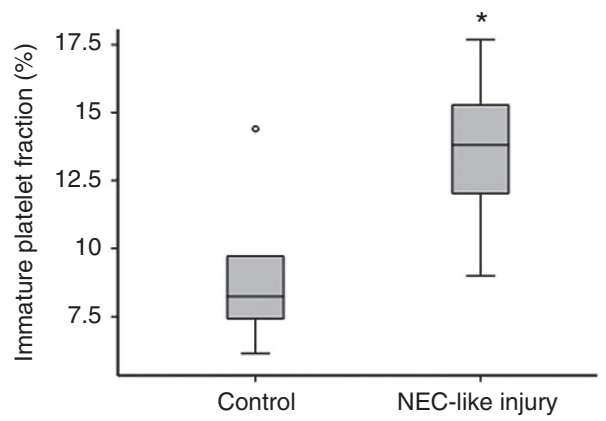

Figure 5. Platelet counts and IPF in P3 mice with TNBS-mediated NEC-like injury. Boxplots show (a) platelet counts and (b) IPF in blood samples from P3 mouse pups in the control vs. NEC-like injury groups $18 \mathrm{~h}$ after TNBS exposure. $N=10$ mice with TNBS-mediated NEC-like injury and five controls. Mann-Whitney $U$-test; ${ }^{*} P<0.05,+P<0.001$ vs. control.

age (gestational age at birth + postnatal age at onset of NEC) of approximately $32 \mathrm{wk}$ (29-31), and therefore, we postulated that the pathoanatomy of NEC may represent a generic injury response of the intestine during a specific developmental epoch and not reflect specific pathogenic trigger(s) (11). In this context, we used TNBS as a nonspecific, but predictable immunological insult to induce bowel injury in P10 C57BL/6 mouse pups. TNBS administration in pups produces an acute inflammatory injury with prominent necrotic changes in distal ileum and proximal colon within $18-24 \mathrm{~h}$, thereby recapitulating the temporal course, regional predilection, and pathoanatomy of human NEC $(11,12)$. The cellular inflammatory response in affected regions is comprised of macrophage-rich infiltrates similar to NEC $(11,12,32)$. There is also a strong similarity in the transcriptional networks activated during TNBS-mediated murine intestinal injury and human NEC (13). TNBS does not cause intestinal injury in germ-free mice, indicating that the mucosal injury requires the presence of intestinal microflora (similar to NEC) and is not due to chemical/corrosive action of TNBS (11).

We recorded a consistent drop in platelet counts in pups with NEC-like injury at 15-24h after TNBS exposure, which is comparable to the onset of thrombocytopenia in most patients within 24-72 h of NEC diagnosis (1-4). Interestingly, platelet counts in P10 mouse pups dropped from baseline values that were three to fourfolds higher than premature human infants 
(33) to their lowest levels of approximately 50, 25, and 12\% in pups with mild, moderate, and severe intestinal injury, respectively. Pups with severe intestinal injury had lowest platelet counts comparable to those in human infants with NEC (1$3,5,6)$. In P3 pups, despite limited scope for kinetic studies with repeated blood sampling or interventions such as intravenous platelet transfusions, TNBS-mediated injury was still informative as the baseline platelet counts were comparable to premature human infants and dropped to nadirs similar to those seen in critically-ill human infants (34). In thrombocytopenic mice, intestinal injury was marked by extensive interstitial hemorrhages in the affected bowel regions similar to surgicallyresected NEC specimens (32), indicating that TNBS-mediated intestinal injury could be a useful preclinical model to study the role of platelets (and platelet transfusions) in NEC.

In our study, murine NEC-like injury was associated with increased platelet volume indices, an early and sustained rise in IPF following TNBS exposure, and increased megakaryocyte number/ploidy in the bone marrow. In pups with moderatesevere injury, we also detected a transient rise in platelet counts at the $6 \mathrm{~h}$ time-point. Taken together, these findings were consistent with increased thrombopoiesis during NEC-like injury. Although we did not investigate the mechanism(s) that may drive platelet production during NEC, low concentrations of lipopolysaccharides are known to stimulate megakaryocyte differentiation and platelet production in conjunction with inflammatory cytokines such as interleukin-6 (35). Our findings are consistent with the observations of Brown et al. (10), who showed a modest increase in circulating reticulated platelets and megakaryocytic precursors in human infants with a diagnosis of NEC. However, in a recent study, Cremer et al. (9) found low IPF in a small cohort of infants with surgical NEC and thrombocytopenia. The reasons for these differences are not clear. One plausible explanation for the dampened thrombopoietic response in some infants with severe NEC may be related to elevated circulating levels of platelet factor 4, which is released from activated platelets and is a potent inhibitor of megakaryocytopoiesis (36).

The detection of increased megakaryocytic commitment/ differentiation and thrombopoiesis during NEC-like injury favors increased peripheral consumption of platelets as the likely mechanism of thrombocytopenia over the alternative possibility of impaired platelet production. The site of platelet consumption in NEC remains to be identified, but is widely presumed to be related to microthrombotic events in the inflamed bowel $(8,37)$. Existing information from animal models indicate that circulating platelets are likely exposed to diverse activators including bacterial products, platelet-activating factor, arachidonic acid metabolites, various factors from the coagulation cascades $(38,39)$. These mediators can stimulate endothelial cells and macrophages to release inflammatory cytokines and nitric oxide, which, along with thromboplastin released from gangrenous bowel, could explain platelet activation and aggregation in the microvasculature $(1,39)$.

In conclusion, we have shown that murine pups with TNBSmediated NEC-like injury develop thrombocytopenia despite evidence of increased megakaryopoiesis and thrombopoiesis. The major strength of our study is the development of a robust preclinical model that could allow the study of NEC-related thrombocytopenia and underlying mechanisms. However, there are also important limitations; animal models may not always capture the complexity of a natural, multifactorial disease process such as NEC. We did not investigate the regulators of thrombopoiesis in this study. Tracing the longitudinal change in endogenous stimulants such as thrombopoietin (Tpo) will be a critical step in understanding whether there is scope for therapeutic stimulation of megakaryocytic precursors with recombinant Tpo or the newer synthetic agonists of the Tpo receptor (40), or if these regulatory systems are already saturated. Finally, studies of NEC-like injury in small animals may overlook physiological covariates such as feeding experience, comorbidities, and microbial flora. Given these limitations, findings in preclinical models need further corroboration from clinical studies.

\section{ACKNOWLEDGMENTS}

The authors would like to acknowledge Sysmex America, Inc. for the instrument loan (Sysmex XT-2000iV), which, at least in part, made this study possible. The authors would like to thank Jolanta Kunicka and Tiffany Ivers for their technical support with the analyzer and useful discussion. They would also like to thank James Palis, MD (University of Rochester) for his review of this manuscript, and to the laboratory of Dr. Martha Sola-Visner (Boston Children's Hospital) for sharing their gating parameters for the identification of mature and immature platelets in the Sysmex XT-2000iV analyzer.

\section{AUTHOR CONTRIBUTIONS}

A.M. and K.N. designed the study and wrote the manuscript, K.N., K.M., L.G., and B.A.T. performed key experiments or data analysis. All the authors contributed to and approved the manuscript.

\section{STATEMENT OF FINANCIAL SUPPORT}

This study was supported by National Institutes of Health, Bethesda, MD, awards HD059142 and HL124078 (to A.M.)

Disclosure: The authors disclose no conflicts.

\section{REFERENCES}

1. O'Neill JA Jr. Neonatal necrotizing enterocolitis. Surg Clin North Am 1981;61:1013-22.

2. Hutter JJ Jr, Hathaway WE, Wayne ER. Hematologic abnormalities in severe neonatal necrotizing enterocolitis. J Pediatr 1976;88:1026-31.

3. Patel CC. Hematologic abnormalities in acute necrotizing enterocolitis. Pediatr Clin North Am 1977;24:579-84.

4. Kenton $\mathrm{AB}$, O’Donovan $\mathrm{D}$, Cass DL, et al. Severe thrombocytopenia predicts outcome in neonates with necrotizing enterocolitis. J Perinatol 2005;25:14-20.

5. Ververidis M, Kiely EM, Spitz L, Drake DP, Eaton S, Pierro A. The clinical significance of thrombocytopenia in neonates with necrotizing enterocolitis. J Pediatr Surg 2001;36:799-803.

6. Ragazzi S, Pierro A, Peters M, Fasoli L, Eaton S. Early full blood count and severity of disease in neonates with necrotizing enterocolitis. Pediatr Surg Int 2003;19:376-9.

7. Miner CA, Fullmer S, Eggett DL, Christensen RD. Factors affecting the severity of necrotizing enterocolitis. J Matern Fetal Neonatal Med 2013;26:1715-9.

8. Sola MC, Del Vecchio A, Rimsza LM. Evaluation and treatment of thrombocytopenia in the neonatal intensive care unit. Clin Perinatol 2000;27:655-79.

9. Cremer M, Weimann A, Szekessy D, Hammer H, Bührer C, Dame C. Low immature platelet fraction suggests decreased megakaryopoiesis in neonates with sepsis or necrotizing enterocolitis. J Perinatol 2013;33:622-6. 


\section{Articles | Namachivayam et al.}

10. Brown RE, Rimsza LM, Pastos K, et al. Effects of sepsis on neonatal thrombopoiesis. Pediatr Res 2008;64:399-404.

11. MohanKumar K, Kaza N, Jagadeeswaran R, et al. Gut mucosal injury in neonates is marked by macrophage infiltration in contrast to pleomorphic infiltrates in adult: evidence from an animal model. Am J Physiol Gastrointest Liver Physiol 2012;303:G93-102.

12. MohanKumar K, Namachivayam K, Chapalamadugu KC, et al. Smad7 interrupts TGF- $\beta$ signaling in intestinal macrophages and promotes inflammatory activation of these cells during necrotizing enterocolitis. Pediatr Res 2016;79:951-61.

13. MohanKumar K, Namachivayam K, Cheng F, et al. Trinitrobenzene sulfonic acid-induced intestinal injury in neonatal mice activates transcriptional networks similar to those seen in human necrotizing enterocolitis. Pediatr Res 2016 doi: 10.1038/pr.2016.189 (Epub ahead of print).

14. Liu ZJ, Hoffmeister KM, Hu Z, et al. Expansion of the neonatal platelet mass is achieved via an extension of platelet lifespan. Blood 2014;123:3381-9.

15. Rubin CI, French DL, Atweh GF. Stathmin expression and megakaryocyte differentiation: a potential role in polyploidy. Exp Hematol 2003;31:389-97.

16. Drachman JG, Sabath DF, Fox NE, Kaushansky K. Thrombopoietin signal transduction in purified murine megakaryocytes. Blood 1997;89:483-92.

17. Schneider CA, Rasband WS, Eliceiri KW. NIH Image to ImageJ: 25 years of image analysis. Nat Methods 2012;9:671-5.

18. Fisher LD, Lin DY. Time-dependent covariates in the Cox proportionalhazards regression model. Annu Rev Public Health 1999;20:145-57.

19. Kruskal W, Wallis WA. Use of ranks in one-criterion variance analysis. J Am Stat Assoc 1952;47:583-621.

20. Daniel WW. Jonckheere-Terpstra test for ordered alternatives. In Daniel WW (ed.). Applied Nonparametric Statistics. PWS-Kent Publishing Company. Boston, 1990. pp. 234-240.

21. Friedman M. The use of ranks to avoid the assumption of normality implicit in the analysis of variance. J Am Stat Assoc 1937;32:675-701.

22. Mann HB, Whitney DR. On a test of whether one of two random variables is stochastically larger than the other. Ann Math Stat 1947;18:50-60.

23. Benjamini Y. Opening the Box of a Boxplot. Am Stat 1988;42:257-262.

24. Abe $\mathrm{Y}$, Wada $\mathrm{H}$, Tomatsu $\mathrm{H}$, et al. A simple technique to determine thrombopoiesis level using immature platelet fraction (IPF). Thromb Res 2006;118:463-9.

25. Sparger KA, Li N, Liu Z, Ramsey H, Sola-Visner MC. Developmental differences between newborn and adult mice in response to romiplostim. Blood 2013;122:3542.

26. Sola-Visner MC, Christensen RD, Hutson AD, Rimsza LM. Megakaryocyte size and concentration in the bone marrow of thrombocytopenic and nonthrombocytopenic neonates. Pediatr Res 2007;61:479-84.
27. Walthall K, Cappon GD, Hurtt ME, Zoetis T. Postnatal development of the gastrointestinal system: a species comparison. Birth Defects Res B Dev Reprod Toxicol 2005;74:132-56.

28. Nanthakumar NN, Dai D, Meng D, Chaudry N, Newburg DS, Walker WA. Regulation of intestinal ontogeny: effect of glucocorticoids and luminal microbes on galactosyltransferase and trehalase induction in mice. Glycobiology 2005;15:221-32.

29. Sharma R, Hudak ML, Tepas JJ 3rd, et al. Impact of gestational age on the clinical presentation and surgical outcome of necrotizing enterocolitis. J Perinatol 2006;26:342-7.

30. Yee WH, Soraisham AS, Shah VS, Aziz K, Yoon W, Lee SK; Canadian Neonatal Network. Incidence and timing of presentation of necrotizing enterocolitis in preterm infants. Pediatrics 2012;129:e298-304.

31. Llanos AR, Moss ME, Pinzòn MC, Dye T, Sinkin RA, Kendig JW. Epidemiology of neonatal necrotising enterocolitis: a population-based study. Paediatr Perinat Epidemiol 2002;16:342-9.

32. Remon JI, Amin SC, Mehendale SR, et al. Depth of bacterial invasion in resected intestinal tissue predicts mortality in surgical necrotizing enterocolitis. J Perinatol 2015;35:755-62.

33. White JR, Gong H, Colaizy TT, Moreland JG, Flaherty H, McElroy SJ. Evaluation of hematologic variables in newborn C57/BL6 mice up to day 35. Vet Clin Pathol 2016;45:87-95.

34. Christensen RD, Henry E, Del Vecchio A. Thrombocytosis and thrombocytopenia in the NICU: incidence, mechanisms and treatments. J Matern Fetal Neonatal Med 2012;25 Suppl 4:15-7.

35. Wu D, Xie J, Wang X, et al. Micro-concentration lipopolysaccharide as a novel stimulator of megakaryocytopoiesis that synergizes with IL-6 for platelet production. Sci Rep 2015;5:13748.

36. Lambert MP, Rauova L, Bailey M, Sola-Visner MC, Kowalska MA, Poncz M. Platelet factor 4 is a negative autocrine in vivo regulator of megakaryopoiesis: clinical and therapeutic implications. Blood 2007;110:115360.

37. Hyman PE, Abrams CE, Zipser RD. Enhanced urinary immunoreactive thromboxane in neonatal necrotizing enterocolitis. A diagnostic indicator of thrombotic activity. Am J Dis Child 1987;141:686-9.

38. Hsueh W, Caplan MS, Qu XW, Tan XD, De Plaen IG, Gonzalez-Crussi F. Neonatal necrotizing enterocolitis: clinical considerations and pathogenetic concepts. Pediatr Dev Pathol 2003;6:6-23.

39. Maheshwari A. Immunologic and hematological abnormalities in necrotizing enterocolitis. Clin Perinatol 2015;42:567-85.

40. Siegal D, Crowther M, Cuker A. Thrombopoietin receptor agonists in primary immune thrombocytopenia. Semin Hematol 2013;50 Suppl 1: S18-21. 